\title{
Investigation of the construction of intelligent logistics system from traditional logistics model based on wireless network technology
}

\author{
Quan Ouyang, Jun Zheng and Shunlin Wang*
}

\begin{abstract}
With the continuous development of science and technology, wireless network technology has played an important role in the construction of intelligent logistics system. Under this background, based on the wireless network technology, the construction of the intelligent logistics system from the traditional logistics model is studied. Through the analysis of the latest wireless network technology, an intelligent logistics system construction algorithm based on this technology is proposed, and the logistics business of traditional logistics model after adopting wireless network technology is designed. The experimental research proves that on the basis of the traditional logistics model, the use of the latest wireless network technology can effectively realize the construction of an intelligent logistics system.
\end{abstract}

Keywords: Wireless network technology, Traditional logistics model, Intelligent logistics

\section{Introduction}

Smart logistics is an inevitable trend in the development of modern logistics [1]. First of all, the concept of low-carbon, environmental protection, green, and resource recyclability is becoming more and more popular in the society, and it is also increasingly used in various industries. More people pay attention to the concept of green logistics and low-carbon logistics in modern logistics [2]. The realization of organizational forms such as integration and flattening, the rapid advancement of Internet of Things technologies, the introduction of management concepts such as industrial linkage and supply chain, all indicate the integration of information, networking, integration, intelligence, flexibility, and agility. Intelligent logistics with features such as visualization and automation are an inevitable trend and ultimate destination of modern logistics development [3]. Secondly, as the pillar industry of modern circulation, the rapid development of e-commerce and chain operations has led enterprises and consumers to rely more and more on modern logistics. The demand for logistics has been unprecedentedly high. Internet company is a new type of flexible sales model. Through a variety of promotional activities, it is setting off a new wave of online shopping among consumers. However, at this stage, the level of China's logistics industry is still far from meeting the high demands of e-commerce and consumers on their efficiency and quality. More energy consumption will be caused, more carbon emissions will created, and huge hidden dangers will be brought to the environment. These are greatly deviating from the era of social energy saving for energy saving and emission reduction. The waste of energy and unreasonable allocation of resources also increase the operating costs of logistics companies and reduce corporate profits [4]. The market's demand for the quantity and quality of logistics, the background of green environmental protection, as well various factors of corporate cost pressures have promoted the development of modern logistics toward intelligence [5].

* Correspondence: rzdz17070@163.com

College of Business Administration, Ningbo Polytechnic, Ningbo, Zhejiang, China 


\section{State of the art}

Wireless sensor network (WSN) is the result of cross-domain interaction between emerging disciplines and traditional disciplines in the field of information science [6]. The technical development of wireless sensor networks has experienced a transition from smart sensors to wireless smart sensors and eventually developed into three phases of wireless sensor networks [7]. The smart sensor stage embeds a chip with data calculation and processing capabilities into the sensor to enhance and extend the working capability of the sensor node. The wireless smart sensor stage adds wireless communication technology to the sensor node based on the smart sensor. The wireless communication capability extends the antenna-awareness of antennae. Wireless sensor networks introduce network technology into wireless smart sensors, which make the sensor no longer a single sensory unit but a network entity capable of exchanging information and coordinating control. The sensor device and the area can be interconnected through nodes, and the data information can be exchanged. Looking at the development of wireless sensor networks, the development of digital wireless network technology determines its progress and application. In the 1970s, the world's first network that broadcasts data packets through satellites-ALOHA network-was established at the University of Hawaii in the United States. Packet (also called cluster) broadcasting is introduced into the wireless channel, creating a new era of digital wireless communication. In order to improve the throughput of ALOHA protocol network system, an improved version called $\mathrm{S}$-ALOHA is proposed. The data sent by the user within the network is unified by the common clock. Then a new carrier sense multiple access media access control (MAC) technology is proposed and the detection of random access multiple access protocols began [8].

\section{Methodology}

\subsection{Intelligent logistics system construction algorithm} based on wireless sensor technology

The four major core radio-frequency identification technologies of the Internet of Things, namely RFID technology, bar code technology, communication technology, remote sensing technology, and the continuous upgrading of intelligent information equipment, are the core driving forces that really promote the rapid development of the Internet of Things. The continuous innovation and development of the Internet of Things has enabled the Internet of Things to have characteristics such as comprehensiveness of perception, reliability of information hulls, and intelligence of information processing. These features have just provided key technical support for the formation and development of smart logistics to achieve logistics information, digitalization, and intelligence. The currently widely used smart logistics system in China is the machine-to-machine framework of machine-to-machine (M2M), and in the samples of grounded theoretic methods, the application layer, service layer, and sensory layer with more frequent words are used. Such words belong to the scope of the M2M architecture. Therefore, the construction of the smart logistics system for this study is planned to adopt this system architecture and combine the characteristics of Yiwu L Group's logistics and distribution to complete the construction of a smart logistics system based on the Internet of Things technology.

The role of the sensory layer is mainly to upload information such as user information, employee information, managerial information, and product information that may be involved in various smart logistics platform personnel, goods, and the environment to the database or server through the Internet of Things technology. The sensory layer in the smart logistics system is mainly composed of the data acquisition layer and the access layer. The data acquisition layer mainly uses the sensors, bar codes, and RFID tags in the Internet of Things technology to capture the information of items under the line to the mobile device or the personal computer (PC) through sensor technology, barcode recognition technology, and radio-frequency identification technology. Then, through the network upload function in the access layer, the network, such as a mobile network, a wireless network, or a wired network, is transmitted to the global IoT network. The design of the network layer is more professional, and most of them involve computer professional knowledge. This article is biased toward theorization, so it can only find relevant supporting materials from the literature and supplement of it with understanding. The network layer in the smart logistics system based on the Internet of Things technology is a bridge connecting customers and background data. Its main function is achieved through the network transmission platform and application platform, and the two platforms can be uniformly understood as an information integration platform. The network layer is the link that connects the major components of the logistics information system and is the basis for realizing information sharing and real-time communication. The basic communication network of logistics information system can be divided into three major components: logistics park network, urban logistics information network, and wireless communication network. The logistics park network serves the logistics management department of the park and the logistics enterprises of the park, connecting their respective management information systems and accessing the 
urban logistics information network to provide timely, smooth, and effective logistics information services for enterprises in the park. Urban logistics information network refers to the urban backbone network that connects the logistics park, logistics public information platform, logistics trading platform, industry management subsystem, and enterprise logistics information system, and realizes the organic integration of information resources of major subsystems. The wireless communication network is the key to realize communication with other logistics information systems such as on-site subsystems, on-board subsystems, vehicle tracking and dispatching systems, etc. It can be established by combining conventional mobile communication technologies with wireless cluster communication technologies.

The application layer designed in this paper includes on-site subsystems, vehicle subsystems, logistics enterprise subsystems, and industry management subsystems. The on-site subsystems are distributed on roads, warehouses, and yards for information collection, provision and industry management subsystems, and logistics. The physical facilities and management systems for information exchange between enterprise subsystems; the vehicle subsystem is composed of information receiving, sending and collecting equipment installed on vehicles, and its main functions are used for information exchange between trucks and data centers; logistics enterprises There are certain differences in the business systems of logistics enterprises under different logistics modes of subsystems. From the perspective of supply chain, the subsystems required by logistics companies are the systems in the functional system; the industry management subsystem is mainly from relevant government departments and logistics. Defined from the perspective of hubs and industry management, its system requirements can meet relatively many functions, and needs to be able to operate with logistics companies, vehicle fleets, logistics transit points, airport station railways, banks, insurance, taxation, and other logistics companies. The intersected departments and enterprises formed information exchanges. No information packet is sent in an idle time slot, that is, the probability of no information packet arriving is:

$$
q^{0}=e^{-a G}
$$

Only one information packet is sent in an idle time slot " $a$," that is, the probability of successfully sending the information packet:

$$
q_{a}^{1}=a G e^{-a G}
$$

The probability of sending no information packets within the transmission period $(1+3 a)$ is:

$$
q_{1+3 a}^{1}=e^{-G(1+3 a)}
$$

The probability of sending only one packet during the transmission period $(1+3 a)$ is:

$$
q_{1+3 a}^{1}=G(1+3 a) e^{-G(1+3 a)}
$$

So, the probability of consecutive $i 1$ and $j$ BU events in one cycle is:

$$
P\left(N_{I}=i, N_{B U}=j\right)=\left(e^{-G a}\right)^{i-1}\left(1-e^{-G a}\right)\left(1-e^{-G(1+3 a)}\right)^{j-1} e^{-G(1+3 a)}
$$

The number of consecutive $I$ events in a cycle $E\left(N_{I}\right)$ is:

$$
\begin{aligned}
E\left(N_{I}\right) & =\sum_{i=1}^{\infty} \sum_{j=1}^{\infty} i P\left(N_{I}=i, N_{B U}=j\right) \\
& =\sum_{i=1}^{\infty} \sum_{j=1}^{\infty} i\left(e^{-G a}\right)^{i-1}\left(1-e^{G a}\right)\left(1-e^{G(1+3 a)}\right)^{j-1} \\
& =\frac{1}{1-e^{-G a}}
\end{aligned}
$$

The number of consecutive occurrences of $j$ BU events in a cycle $E\left(N_{B U}\right)$ is:

$$
\begin{aligned}
E\left(N_{B U}\right) & =\sum_{i=1}^{\infty} \sum_{j=1}^{\infty} i P\left(N_{I}=i, N_{B U}=j\right) \\
& =\sum_{i=1}^{\infty} \sum_{j=1}^{\infty} i\left(e^{-G a}\right)^{i-1}\left(1-e^{G a}\right)\left(1-e^{G(1+3 a)}\right)^{j-1} e^{G(1+3 a)} \\
& =\frac{1}{1-e^{-G(1+3 a)}}
\end{aligned}
$$

In the $\mathrm{BU}$ event, the number of successful events $U$ is as follows. Before the analysis, the following definitions are made: $U$ : During the idle period, when the packet arrives in the last slot, and only one packet arrives, the packet is successfully transmitted in the next slot. It can be known that the throughput of the traditional slotted non-persistent Carrier Sense Multiple Access (CSMA) protocol is:

$$
S=\frac{G a e^{-G a}}{1-e^{-G a}+a}
$$

\subsection{Logistics business system construction design model}

The logistics business system is centered on transportation, warehousing, distribution, information, packaging, distribution processing, loading and unloading, and transportation. Meanwhile, it is supported by relevant factors that ensure the operation of the logistics business, such as advanced logistics facilities and equipment, and modern operation modes, to achieve high efficiency and low consumption logistics activities (Fig. 1). Among them, 


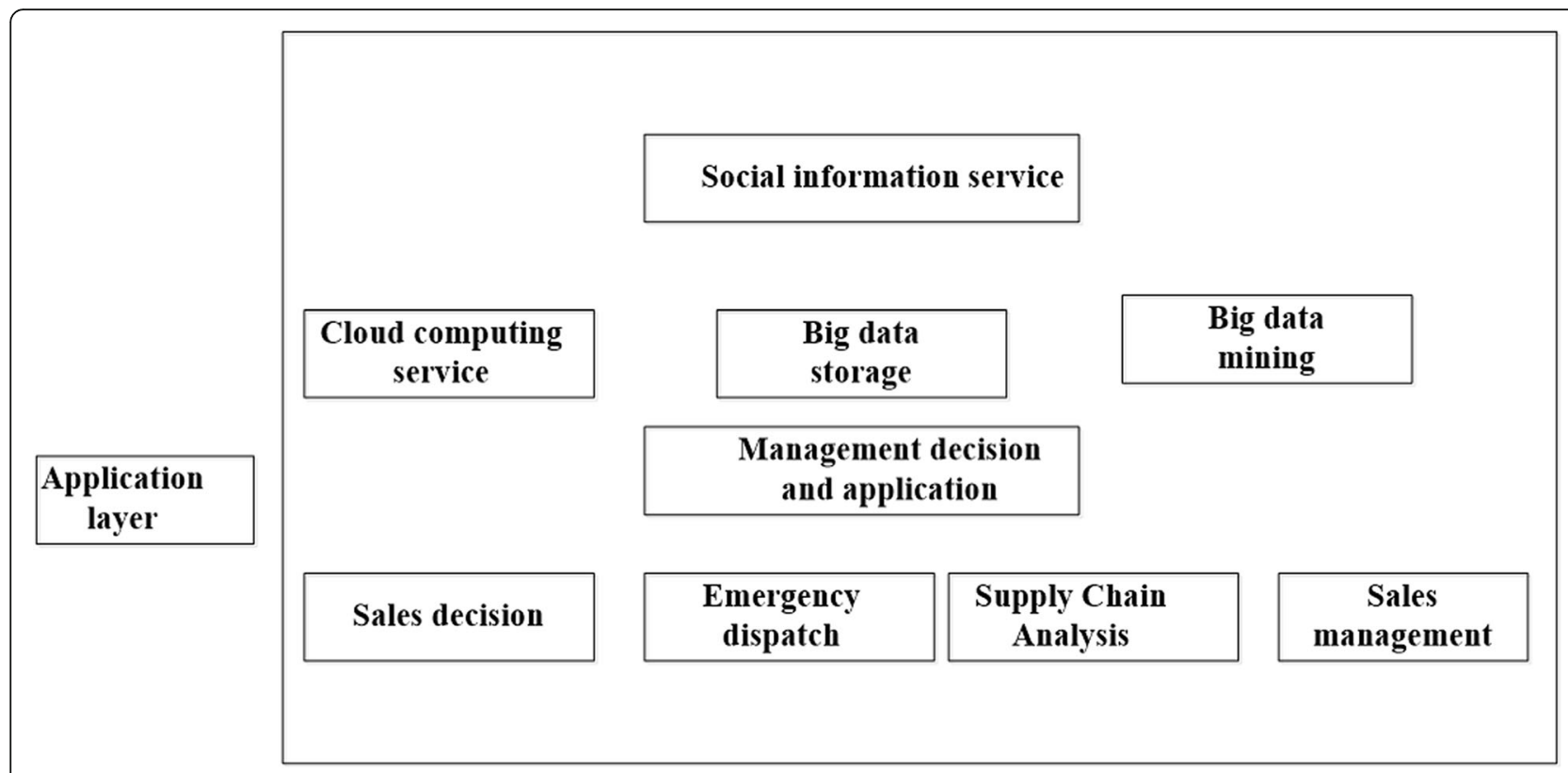

Fig. 1 Smart logistics system architecture

transportation, warehousing, and distribution are the most basic and important businesses of logistics. Information is the chain linking business activities in all aspects of logistics, and it is also an important means to carry out and complete the logistics business. It is the core business layer in the logistics business system. In the traditional logistics business system, real-time monitoring and control of objects cannot be achieved due to limited information collection and interaction capabilities. Based on the internet of things technology, the logistics business system, the comprehensive perception, reliable transmission, and intelligent processing of the internet of things create the basic conditions for the transparency and real-time management of goods, and the logistics traceability management of important goods. They will certainly improve the intelligentization of logistics business system, which will bring changes to the logistics business system and processes. The framework of the logistics business system based on the Internet of Things is shown in Fig. 2.

In Fig. 3, there are four processes: A, B, C, and D, and each process details are as follows: Only one information packet arrives, and the next time slot is transmitted successfully. $\mathrm{B}$ and $\mathrm{C}$ have more than two information packets arriving, the next time slot conflicts, and the conflict is resolved in the subsequent idle period. In the process of conflict resolution, newly arrived information packets adopt non-independent CSMA. After the CSMA is decomposed, the CSMA continues to use the non-consolidation

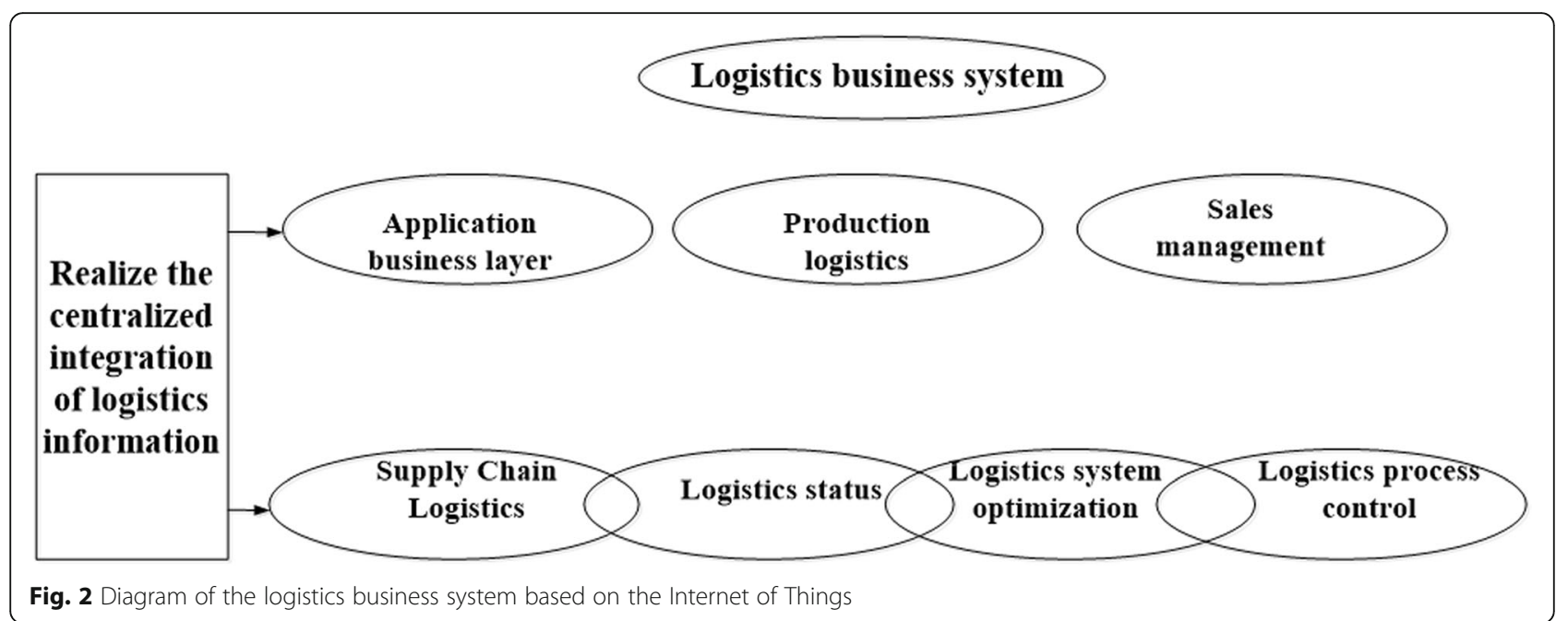




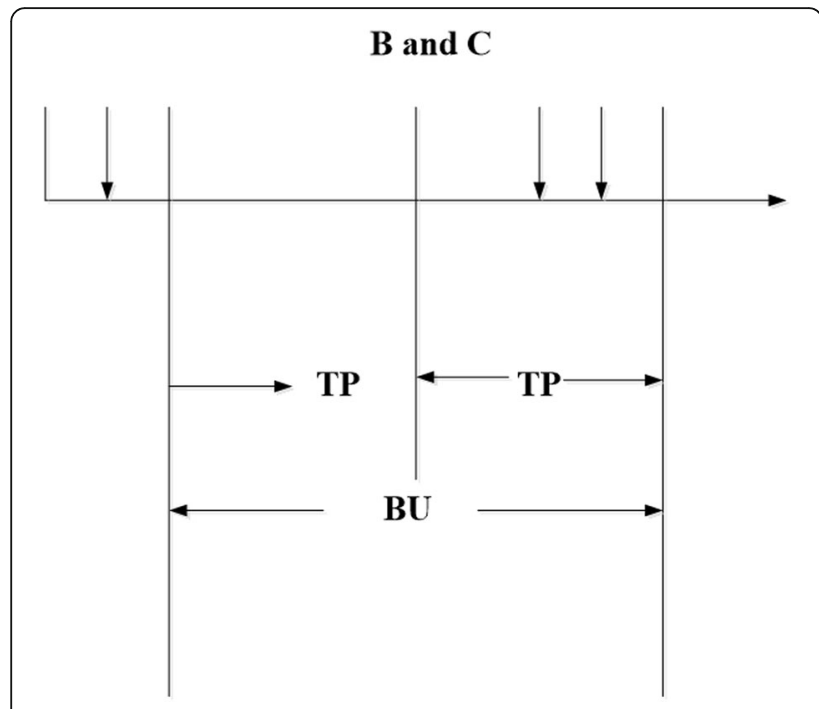

Fig. 3 Non-independent CSMA protocol model based on binary tree conflict resolution with monitoring function

protocol based on binary tree conflict resolution with monitoring functions. After the introduction of the improved binary tree conflict resolution algorithm, the effective time slot length occupied by the successful transmission of information packets in one cycle is:

$$
E\left(U^{*}\right)=E\left(N_{U}\right) \times(1+3 a)=\frac{G a e^{-G a}(1+3 a)}{1-e^{-G a}}
$$

The throughput of non-intrusive CSMA protocol with monitoring function based on binary tree conflict resolution is:

$$
S=\frac{E\left(U+E\left(U_{B x}\right)\right.}{E\left(U^{*}\right)+E\left(U_{B x}\right)+E(I)}
$$

\section{Result analysis and discussion}

In terms of system architecture and function system, Yiwu Group L has built several supporting main systems including logistics data exchange center, communication infrastructure network, field subsystem, vehicle subsystem, logistics enterprise subsystem, and industry management subsystem. And using various systems to build various types of information platforms, service platforms, and trading platforms to achieve the ability of users to achieve on-line completion of inquiries, orders, payments, and other operations. In the construction of the support system, it coordinated the investment and construction of major projects, gave full play to the coordination intelligence of logistics-related government departments, the formulation intelligence of standards and regulations, the intelligence of investment management, and the intelligence of the construction of smart logistics platforms; in terms of technical conditions, Yiwu L Group has used foreign capital to introduce advanced Internet of Things technologies and long-range facilities and equipment, and learned to formulate relevant industry standards; in terms of capital markets, the maintenance, operation, and promotion of logistics public information platforms, logistics trading platforms, etc. Neutral information service providers in specific logistics companies are responsible for the government's investment in infrastructure construction, the use of paid fees, and the government's participation in the management of charges. The charging costs should be aimed at maintaining the daily operations of information service providers; in terms of human resources, Yiwu L Group has a professional team that is both familiar with logistics management and computer and communication technology to assist in the planning, guidance, and organization of R\&D and construction of logistics information systems. The cultivation of logistics informatization talents Yiwu City and L Group also cooperated with relevant universities to form a better school-enterprise cooperation mechanism. Through logistics and informatization professional technical training, the talent gap of logistics informatization is filled and the construction of Yiwu logistics information system is ensured.

In the specific network transmission, various types of information collected during the process of logistics awareness are collectively transmitted to the information processing center. The information processing center selects and corrects basic logistics information, completes the transformation of different information, and finally passes the processed logistics information through the transmission network. To the logistics application layer, to achieve inter-regional network interoperability and high-speed transmission of information, to achieve the sharing of local area network resources, centralized information processing, to achieve logistics, vehicle loading and dispatch management intelligence, visualization, and automation. Logistics applications adopts technologies such as cloud computing and data warehouse to centrally integrate and effectively handle logistics information resources, forming a dynamic data resource library that adapts to the needs of each logistics business and is updated in real time; and the logistics status are completed through the management and control of information integration. The real-time monitoring provides a unified information resource support for the development of logistics business and realizes the connection with various logistics business applications; finally, it displays effective information to users, and provides services for the intelligent control of the logistics business of the IoT environment.

The conclusion is drawn that in Fig. 4, the simulated values of the throughput of the CSMA with 


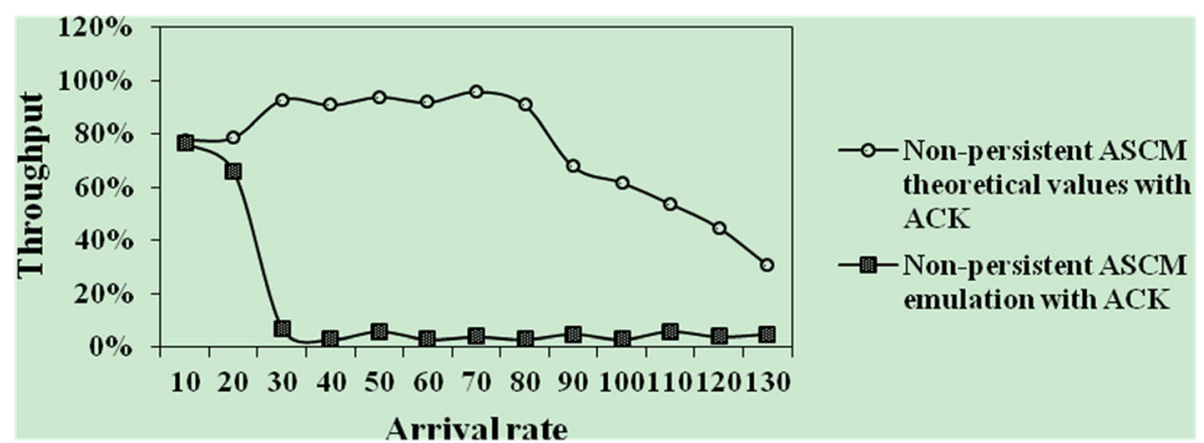

Fig. 4 Comparison of simulated and theoretical values of non-independent CSMA with monitoring function and traditional one-slot nonindependent CSMA throughput when $a=0.01$

monitoring function and the traditional CSMA protocol are consistent with the theoretical values. Due to the fact that more network resources are occupied, when the value of " $a$ " is the same, the throughput of non-independent CSMA with monitoring function is smaller than that of the traditional CSMA protocol. In Fig. 5, the simulated value of the new protocol throughput is consistent with the theoretical value. When the value is getting smaller and smaller, the throughput of unconstrained CSMA protocol with monitoring function based on binary tree conflict decomposition is increasing. In Fig. 6, the simulation values of the three protocol throughput values are consistent with the theoretical values. When the value of $a$ is the same, compared with the non-independent CSMA protocol with monitoring function based on the binary tree conflict resolution and the non-independent CSMA with monitoring function, the throughput of the new protocol has been significantly improved.

\section{Conclusion}

The research on China's smart logistics is still relatively lacking. Most of them remain in the research of a certain technology or some link in the smart logistics system. There is no systematic research on the research of the smart logistics, and the study of the smart logistics system is basically a blank. The relevant literature of the smart logistics system based on the Internet of Things technology is first reviewed, and the concept of Internet of Things technology and smart logistics is defined. The smart logistics system is described, and the relevant structure and characteristics of smart logistics are introduced. Secondly, through the grounding of the theoretical method, the subdivision into system construction, function system construction, and support system construction are completed to construct a smart logistics system. Through previous data research and through a theoretical sampling method, relevant research on China's smart logistics development in China National Knowledge Infrastructure (CNKI) is collected, and the data is sampled and processed. Through the process of data analysis, such as open decoding, spindle decoding, selective decoding and other three decodings, and the selected data is systematically studied, and corresponding concepts are obtained and classified to

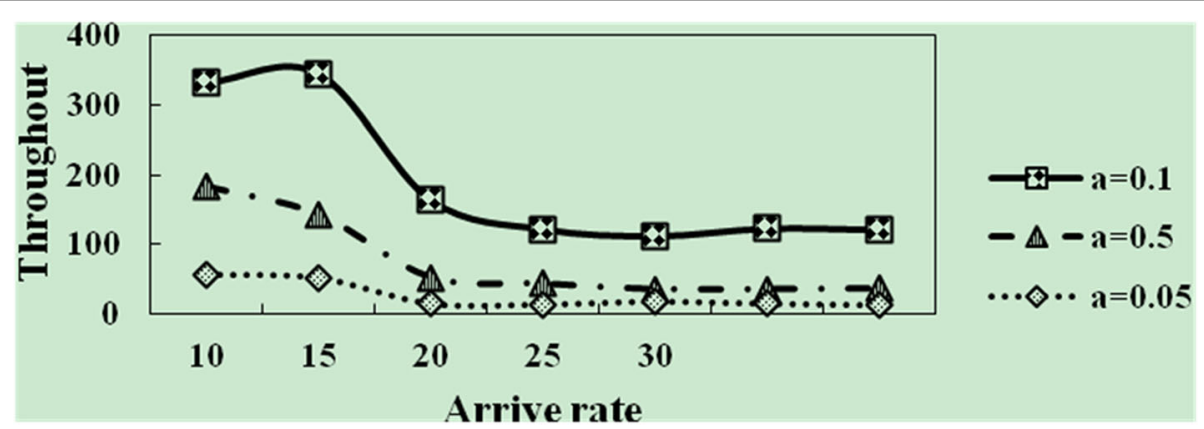

Fig. 5 Comparison of simulation throughput and theoretical values of new protocol throughput when $a=0.01, a=0.1$, and $a=0.5$ 


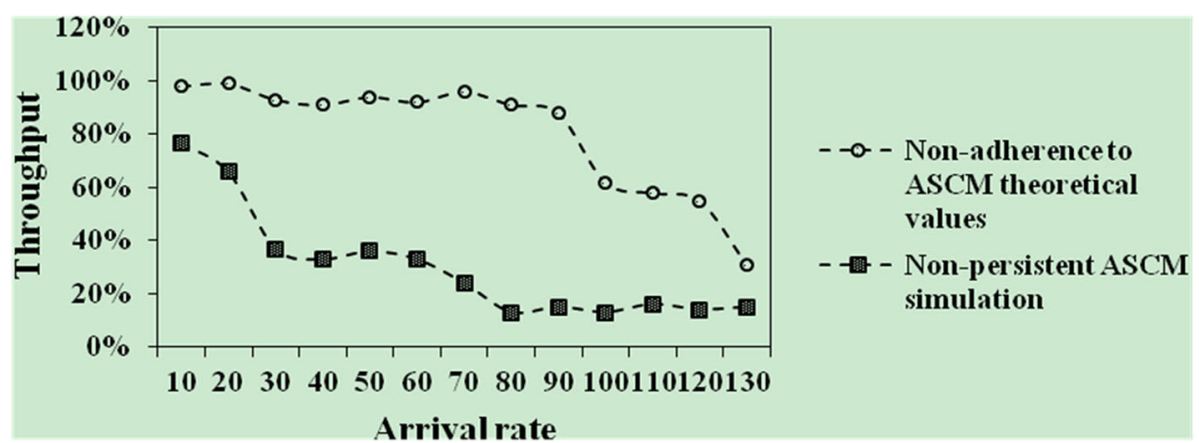

Fig. 6 Comparison of the simulated and theoretical values of three types of protocol when $a=0.01$

form categories. In addition, different categories are classified and analyzed and the theoretical saturation is tested.

\section{Abbreviations}

ACK: Acknowledgement; CNKI: China National Knowledge Infrastructure CSMA: Carrier Sense Multiple Access; M2M: Machine-to-machine; MAC: Media access control; PC: Personal computer; RFDI: Radio-frequency identification

\section{Funding}

The study was supported by "The humanities and Social Sciences Subject of the Ministry of Education Project, Beijing, China (Grant No.16YJA630054) and "The key research base of the philosophy and Social Sciences in Zhejiang province, Zhejiang, China (Grant No.15JDXX01YB)".

\section{Authors' contributions}

QO has made great contributions to the wireless network technology logistics system. JZ has made a great contribution to the data of traditional logistics. SW has made great contributions to the summary and data analysis of this article on intelligent logistics system. All authors read and approved the final manuscript.

\section{Authors' information}

Quan Ouyang, Doctor of Economics, Associate professor. Graduated from Southwestern University of Finance and Economics in 2008. Worked in Ningbo Polytechnic. His research interests include Logistics management and Circulation economy.

Jun Zheng Doctor of Management, Lecturer. Graduated from University of Shanghai for Science and Technology in 2017. Worked in Ningbo

Polytechnic. His research interests include Management Science and Engineering.

Shunlin Wang Master of Management, Professor. Graduated from Jilin University in 2002. Worked in Ningbo Polytechnic. His research interests include Logistics information technology.

\section{Competing interests}

The authors declare that they have no competing interests.

\section{Publisher's Note}

Springer Nature remains neutral with regard to jurisdictional claims in published maps and institutional affiliations.

Received: 3 November 2018 Accepted: 21 December 2018 Published online: 24 January 2019

\section{References}

1. X. Sun, W. Zhao, B. He, et al., Design and research on the logistics internet of vehicles application layer based on wireless network. Electron. Measur. Technol 47(2), 568-832 (2016)

2. W.Y. Zheng, Intelligent logistics packaging system design of the piano. Packag. Eng 4(2), 4568-4722 (2017)
3. J. Xing, An intelligent logistics tracking system based on wireless sensor network. Int. J. Online Eng. 14(1), 17-73 (2018)

4. L.I. Ya-Ya, L. Wang, Y.T. Peng, The intelligent development of logistics industry and the improvement of its performance - an empirical test based on WIOD data and regression model. China Bus. Mark. 8(1), 568-622 (2018)

5. T. Zhang, X. Wu, Research on intelligent logistics development model based on internet of things and cloud computing in big data age. Revista De La Facultad De Ingenieria 32(6), 341-346 (2017)

6. L. Liu, Construction and application analysis of internet of things in intelligent logistics management. Logist. Eng. Manag 7(2), 679-722 (2018)

7. Y. Rong, L.I. Bing, H.U. Yan, An experimental study for intelligent logistics: a middleware approach. Chin. J. Electron. 25(3), 561-569 (2016)

8. S.U. Yufeng, Research on application of RFID technology in intelligent logistics. J. Henan Institute Educ. 67(4), 5943-6125 (2017)

\section{Submit your manuscript to a SpringerOpen ${ }^{\circ}$ journal and benefit from:}

- Convenient online submission

- Rigorous peer review

- Open access: articles freely available online

- High visibility within the field

- Retaining the copyright to your article

Submit your next manuscript at $\boldsymbol{\nabla}$ springeropen.com 